\title{
In silico Drug Repurposing of FDA-Approved Artemisinins as Potent Chemotherapeutics Targeting Bcl-2, CDK-6 \& VEGFR-2: Density Functional Exploration and Molecular Docking Study
}

\author{
Shazia Parveen $1, *$ (D) \\ 1 Faculty of Science, Chemistry Department, Taibah University, Yanbu Branch, 46423, Yanbu, Saudi Arabia \\ * Correspondence: shazia021@gmail.com;
}

Scopus Author ID 57187913400

Received: 20.08.2020; Revised: 11.09.2020; Accepted: 12.09.2020; Published: 15.09.2020

\begin{abstract}
The strategy of using existing drugs originally developed for one disease to treat other indications has found success across medical fields. This paper focuses on drug repurposing of artemisinin and its derivatives (artenimol, artemether, artemotil, and artesunate) that kill malaria parasites for anticancer agents, specifically targeting Bcl-2, CDK-6, and VEGFR-2. The Artemisinins 1-5 were analyzed for compliance with Lipinski's drug-likeness rule and optimum ADME parameters. The results of which revealed all calculated physicochemical descriptors and pharmacokinetic properties are within the expected thresholds. Toxicity in terms of predicted median lethal dose (LD50) in $\mathrm{mg} / \mathrm{kg}$ weight of investigated Artemisinins 1-5 is also reported. Artemisinins 1-5 were subjected to molecular docking and Density Functional Theory (DFT) analysis to discern their molecular interactions at the active site of Bcl-2, CDK-6, and VEGFR-2. The molecular docking study revealed that Artemisinins 1-5 were able to target CDK-6 and VEGFR-2. DFT/B3LYP theoretical calculations for optimization, DFT, frequency, and HOMO/LUMO were performed to obtain electronic and structural properties, chemical reactivity descriptors. Hence, these findings will be highly beneficial in optimizing the utility of the development of Artemisinins 1-5 for cancer therapeutics, specifically targeting CDK-6 and VEGFR-2.
\end{abstract}

Keywords: Artemisinins; VEGFR-2; ADME; molecular docking; DFT.

(C) 2020 by the authors. This article is an open-access article distributed under the terms and conditions of the Creative Commons Attribution (CC BY) license (https://creativecommons.org/licenses/by/4.0/).

\section{Introduction}

According to WHO's global cancer profile, 9.5 million deaths have occurred worldwide due to cancer (www.paho.org). Cancer, also known as a malignant tumor, is a disease described by the unrestrained growth and spread of abnormal cells without any boundary in an organism [1]. Although the origin of cancers is due to genetic adaptations, it could be due to any of these factors- i) activation of oncogenes, ii) inactivation of tumor suppressor genes, iii) inactivation of genes responsible for apoptosis, and iv) mutations produced by chemical, physical and biological agents and are characterized by functional loss owing to the absence of differentiation, uncontrolled proliferation, invasiveness of adjacent tissues and metastasis [2]. The mechanism of cancer is still not understood completely. Currently available anticancer drugs show poor selectivity causing cytotoxicity for dividing 
cells leading to serious side effects, such as immunosuppression, anemia, diarrhea, nausea, and alopecia [3]. In addition, the acquired resistance of different cancer types is a major drawback to cancer treatment strategies [4]. These factors necessitate the discovery of new, more active, and more selective anticancer drugs.

The process for new drug development, preclinical research, and approval are expensive, and time-taking can take up to a decade. This lengthy discovery process unlocks the doors for drug repurposing (also known as drug repositioning, drug reprofiling, indication expansion, or indication shift) as an alternative approach for cutting down the time required to develop a drug [5]. Even though this approach is not new, it has gained substantial impetus in the last decade: about one-third of the approvals in recent years correspond to drug repurposing, generating around $25 \%$ of the annual revenue for the pharmaceutical industry [6]. Pharmacophore-based techniques are nowadays an important part of many computer-aided drug design workflows and have been successfully applied for tasks such as virtual screening, lead optimization, and de novo design [7].

Keeping this in view, current work aims at the drug repurposing approach of already approved anti-malarial Artemisinins 1-5, for exploring their anticancer potential. Artemisinin 1 is a potent anti-malarial drug isolated from the plant Artemisia annua, and its bioactive derivatives have been semi-synthesized, including artenimol (dihydroartemisinin) 2, artemether 3, artemotil 4, and artesunate 5 [8] (Figure 1). In addition to its well established anti-malarial properties, compelling evidence has emerged in the field, showing that artemisinin-derived compounds have potent anticancer activities in a variety of human cancer cell types [9].

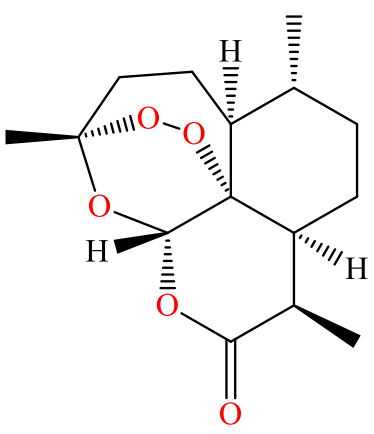

Artemisinin

1

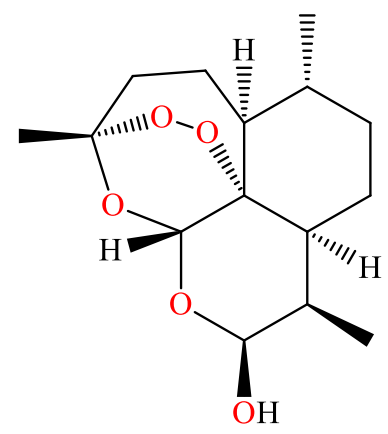
Artenimol 2

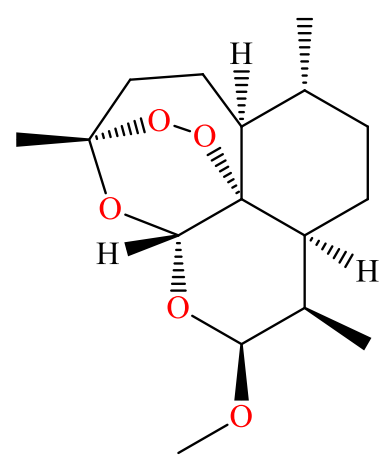

Artemether
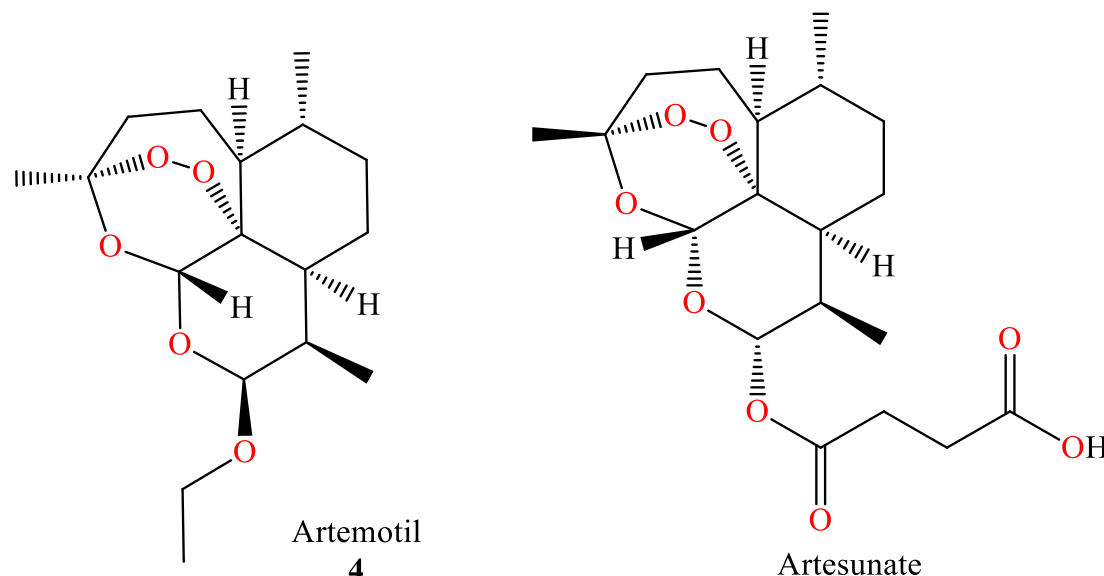

Artesunate

5

Figure 1. Structures of anti-malarial Artemisinins 1-5 used in this study. 
Cancer is triggered by diverse pathways, encompassing several enzymes, most common enzymes that are involved in the cancer development are Bcl-2, Cyclin-dependent kinase-6 (CDK-6), and Vascular Endothelial Growth Factor Receptor-2 (VEGFR-2) [10,11]. BCL-2 family of proteins plays a key role in apoptotic (programmed cell death) regulation [12-15]. Evasion of apoptosis is vital to the pathogenesis of cancer. While on the other hand, cyclindependent kinases (CDKs) functions in cell-cycle progression, transcriptional regulation, DNA damage repair, stem-cell self-renewal, metabolism, spermatogenesis, and neuronal function [16-18]. CDK-6 is a cell-cycle kinase that is responsible for the regulation of the exit from the G1 phase. It has been described as 'central node' in the organization of cell signaling pathways, ultimately lead to tumor development [19-22]. Angiogenesis is a process of generation of new blood vessels from pre-existing ones and is an essential physiological process for solid tumor cell propagation by providing oxygen and nutrients to the tumor cells to enhance their growth and metastasis [23,24]. VEGFR-2 is a tyrosine kinase receptor expressed in endothelial cells [25]. VEGFR-2 plays an important role in anti-angiogenesis and is an effective target for inhibiting tumor cell proliferation and metastasis [26,27].

Considering the above research findings, the current work focuses on the drug repurposing of anti-malarial Artemisinins 1-5 for cancers specifically targeting BCl-2, CDK6, and VEGFR-2 via molecular docking approaches. The outcome of this study will help in optimizing the use of Artemisinins 1-5 as anticancer drugs by contributing to the understanding of its yet unexplored molecular mechanisms of actions.

\section{Materials and Methods}

\subsection{In silico ADME, drug-likeness, and toxicity.}

In silico screening of pharmacological properties (ADME) and drug likeliness of the investigated drugs were performed by SwissADME (http://www.swissadme.ch/index.php) [28]. The toxicity was predicted using ProTox-II chemical toxicity predictions (http://tox.charite.de/protox_II/) [29]. The analysis of different descriptors viz., calculated octanol/water partition coefficient, molecular weight, molecular volume, and a number of hydrogen bond donor and acceptor groups of all the drugs revealed that their penetrating ability in the biological membranes, as determined by the Lipinski rule of five [30]. Computational analyses to predict the core pharmacokinetics parameters such as blood-brain barrier (BBB) permeability, gastrointestinal (GI) absorption, P-glycoprotein-mediated efflux (Pgp) were also performed.

\subsection{Molecular docking.}

The molecular docking studies were performed by using AutoDock Vina [31]. The crystal structures of target proteins Bcl-2 (PDB ID: 2O2F) [32], CDK-6 (PDB ID: 1XO2) [33] and VEGFR-2 (PDB ID: 4ASD) [34] were downloaded from the Protein Data Bank (http://www.rcsb.org/pdb) in PDB format and were prepared by AutoDock Tools [35]. Visualization of the docked pose has been done by using CHIMERA (www.cgl.ucsf.edu/chimera) and Discovery Studio visualizer.

\subsection{DFT calculations.}

Density functional theory (DFT) has been proved to be a powerful tool for the study of 
electronic and thermodynamic parameters. The quantum computational studies of Artemisinins 1-5 were performed in the gas phase on GAMESS [36,37]. Considering the complexity of the theoretical model used for this study, all the structural optimizations were carried out at the DFT using a basis set of B3LYP/3-21G method. The electronic properties of the drugs such as Eномо, Elumo, HOMO-LUMO energy gap, global hardness, electronegativity, electronic chemical potential, electrophilicity, and chemical softness, natural charges, and dipole moment were calculated [38-40].

\section{Results and Discussion}

\subsection{In silico ADME, drug-likeness, and toxicity.}

The major parameters for pharmacokinetics are absorption, distribution, metabolism, and excretion [41]. The predicted Lipinski's parameters molecular weight (MW), number of rotatable bonds (nrotb), number of hydrogen bond acceptors (nON), number of hydrogen bond donors (nOHNH), and lipophilicity (mLogP) and topological polar surface area (TPSA) for investigated drugs are shown in Table 1.

Table 1. Selected calculated physicochemical and pharmacokinetic properties of Artemisinins 1-5.

\begin{tabular}{c|c|c|c|c|c|c|c|c|c|c|c}
\hline Drug & $\mathbf{m L o g P}$ & $\begin{array}{c}\text { TPSA } \\
\left(\AA^{\mathbf{2}}\right)\end{array}$ & $\begin{array}{c}\mathbf{M W} \\
(\mathbf{g} / \mathbf{m o l})\end{array}$ & $\mathbf{n H B A}$ & $\mathbf{n H B D}$ & $\begin{array}{c}\mathbf{n} \\
\text { violations }\end{array}$ & Nrotb & $\begin{array}{c}\text { GI } \\
\text { absorbtion }\end{array}$ & $\begin{array}{c}\text { BBB } \\
\text { permeant }\end{array}$ & $\begin{array}{c}\text { Pgp } \\
\text { substrate }\end{array}$ & LogS \\
\hline $\mathbf{1}$ & 2.62 & 53.99 & 282.33 & 5 & 0 & 0 & 0 & High & Yes & No & -3.42 \\
\hline $\mathbf{2}$ & 1.95 & 57.15 & 284.35 & 5 & 1 & 0 & 0 & High & Yes & No & -3.49 \\
\hline $\mathbf{3}$ & 2.60 & 46.15 & 298.37 & 5 & 0 & 0 & 1 & High & Yes & No & -3.85 \\
\hline $\mathbf{4}$ & 2.85 & 46.15 & 312.40 & 5 & 0 & 0 & 2 & High & Yes & No & -4.10 \\
\hline $\mathbf{5}$ & 1.88 & 100.52 & 384.42 & 8 & 1 & 0 & 5 & High & No & No & -3.08 \\
\hline
\end{tabular}

mLogP: lipophilicity; TPSA: Total Polar Surface Area; MW: Molecular Weight; nHBA: number of hydrogen bond acceptors; nHBD: number of hydrogen bond donors; $n$ violations: number of violated drug-likeness rules; nrotb: number of rotating bonds, BBB: blood-brain barrier; GI: gastrointestinal; Pgp: p-glycoprotein; LogS: solubility

The results of in silico properties of all five Artemisinins 1-5 suggests that there are no significant violations of Lipinski's rule of five $(\mathrm{mLogP}<4.15, \mathrm{MW}>500$, Hydrogen bond donors $<5$ and Hydrogen bond acceptors $<10$ ) [30], since all calculated physicochemical descriptors and pharmacokinetic properties are within the expected thresholds. TPSA is measured the bioavailability of the drug molecule and closely related to the hydrogen bonding potential and should be $<160 \AA$ [42]. According to this model, the drugs showed satisfactory oral bioavailability in combination with lipophilicity, MW, polarity, solubility, saturation, flexibility in an acceptable range, as shown in radar plots [43]. Drug-likeness was determined by the number of free rotatable bonds and Lipinski's rule. Computational analyses to predict the core pharmacokinetics parameters such as gastrointestinal absorption, P-glycoproteinmediated efflux was also performed, and results are displayed in Table 1.

The bioavailability radar of Artemisinins 1-5 provided from SwissADME displayed that they exhibit promising predicted physicochemical properties for oral bioavailability (Figure 2). The ideal space of six physicochemical parameters, for example, size, polarity, lipophilicity, solubility, flexibility, and saturation for oral bioavailability, are located in the pink-colored area $[43,44]$. All the five investigated Artemisinins 1-5 are present in the pink area. Prediction of ligand-based target highly efficient and fast in predicting correct protein targets of compounds in drug discovery $[45,46]$. 


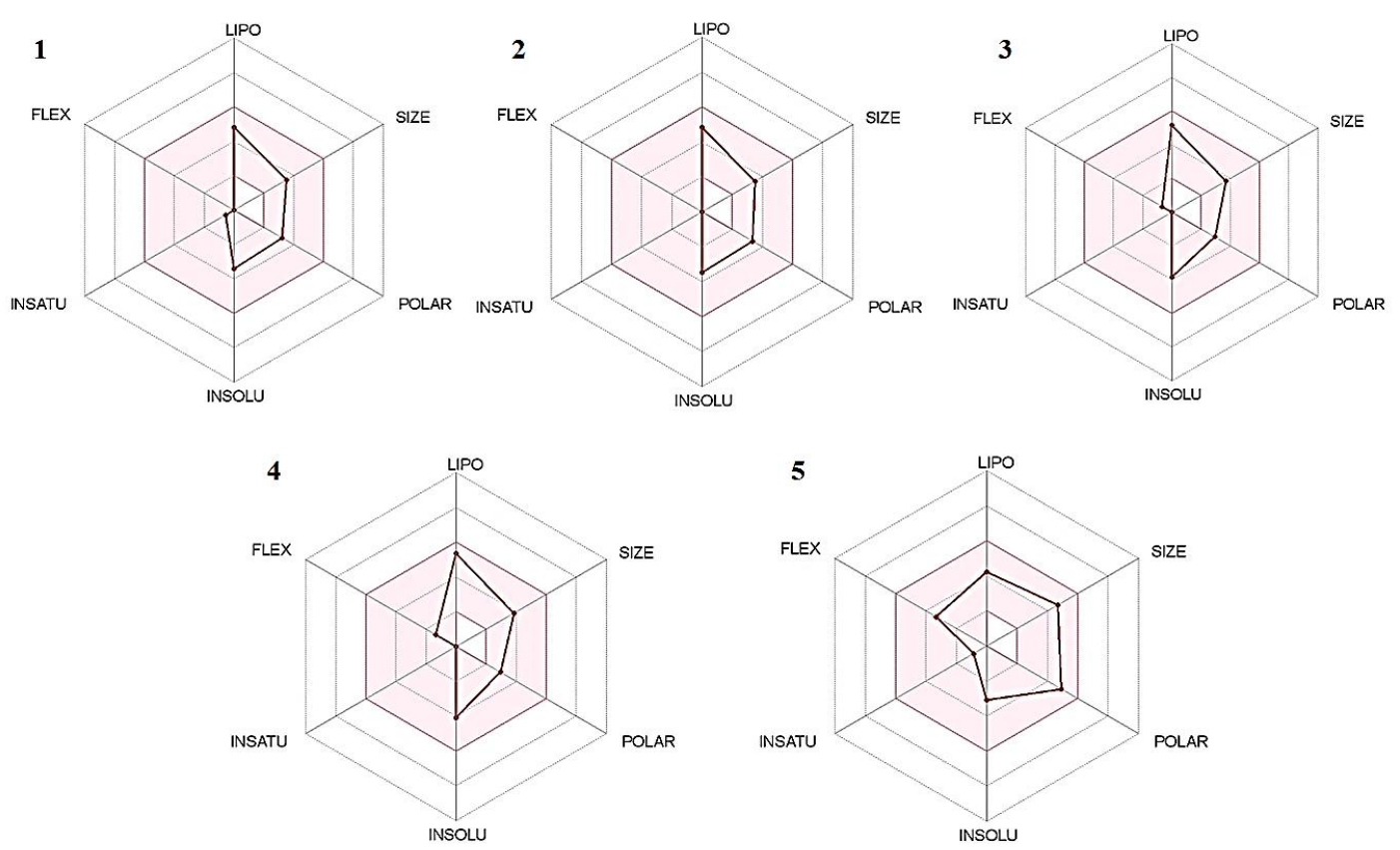

Figure 2. Bioavailability radar plot of Artemisinins 1-5. POLAR (polarity), LIPO (lipophilicity), INSOLU (solubility), FLEX (flexibility), and INSATU (saturation).

Toxicity in terms of predicted median lethal dose (LD50) in $\mathrm{mg} / \mathrm{kg}$ weight of investigated Artemisinins 1-5 is given in Table 2. ProTox-II methods have the potential to support risk assessments for regulatory decisions, such as to create novel hypotheses and get insights into the mechanisms of toxicity [29].

Table 2. Predicted $\mathrm{LD}_{50}(\mathrm{mg} / \mathrm{kg})$ to represent compound toxicity of Artemisinins 1-5.

\begin{tabular}{c|c} 
Drugs & LD 50 (Toxicity Class) \\
\hline $\mathbf{1}$ & $4228(5)$ \\
\hline $\mathbf{2}$ & $567(4)$ \\
\hline $\mathbf{3}$ & $567(4)$ \\
\hline $\mathbf{4}$ & $567(4)$ \\
\hline $\mathbf{5}$ & $1000(4)$ \\
\hline LD50: Half-maximal (50\%) lethal dose
\end{tabular}

\subsection{Molecular docking.}

The Docking studies were performed to study the molecular binding pattern of Artemisinins 1-5 within the active pocket of the crystal structures of the anticancer targets. The targets used for docking analysis with Artemisinins 1-5 are Bcl-2, CDK-6 and VEGFR-2 which have been widely indicated to contribute in apoptotic regulation, cell-cycle progression, transcriptional regulation, DNA damage repair, stem-cell self-renewal, metabolism, spermatogenesis and neuronal function and in anti-angiogenesis $[12,16,17,19,20,23,24,26,27,47]$. The binding of the ligand in the active site of a target is indicative of the probability that the ligand may possibly be capable of steering the functional alteration of the target molecules [48,49]. Drug-target interactions were also decoded in terms of interacting amino acid residues, hydrogen bonding, docking energy analysis, and comparisons of active site amino acid residues and probable binding sites. The docking analysis of Artemisinins 1-5 within the active binding sites of targets is shown in Table 3. 
Table 3. Molecular docking analysis of Artemisinins 1-5 within the binding sites of Bcl-2, CDK-6, and VEGFR-2.

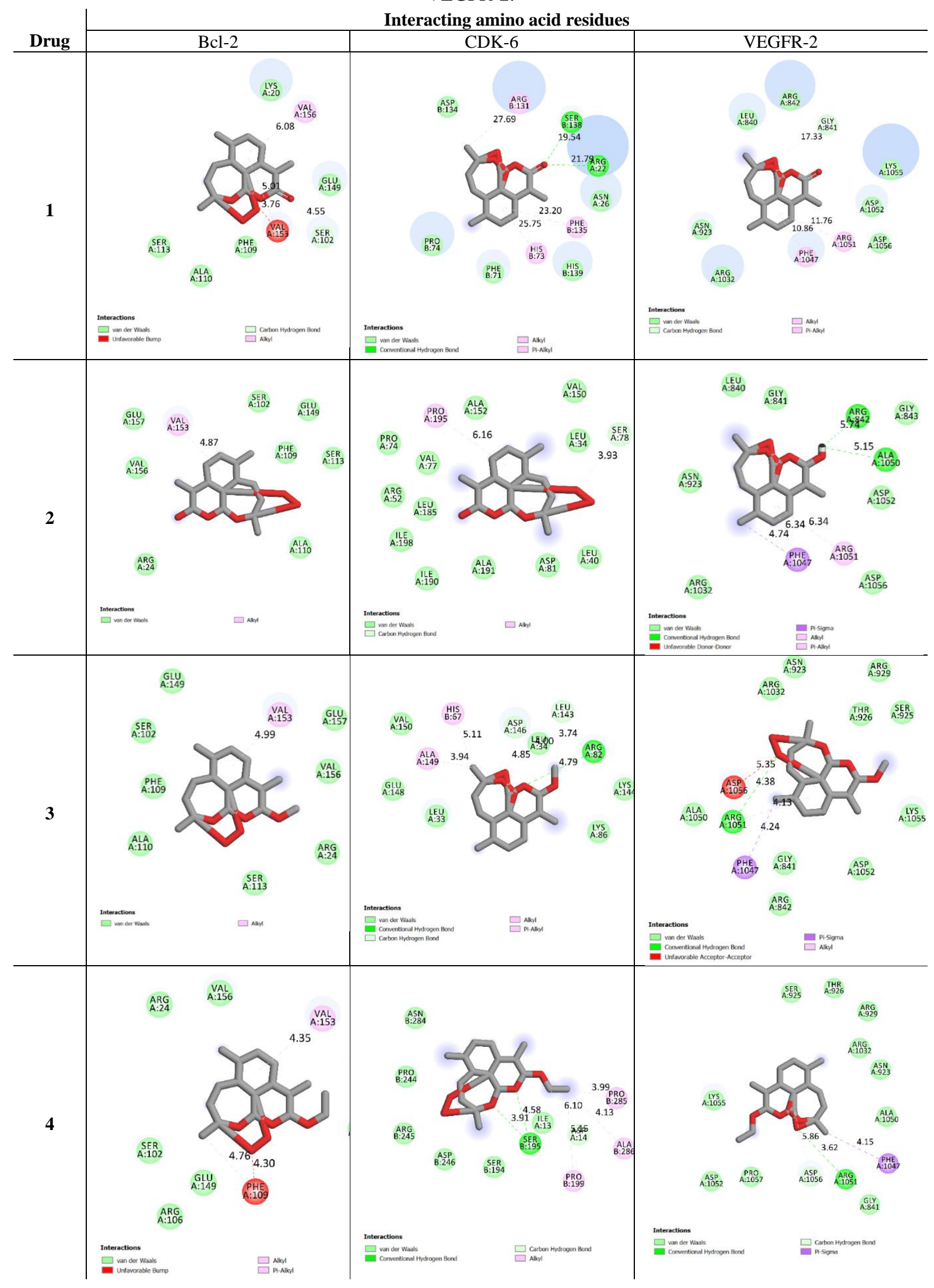




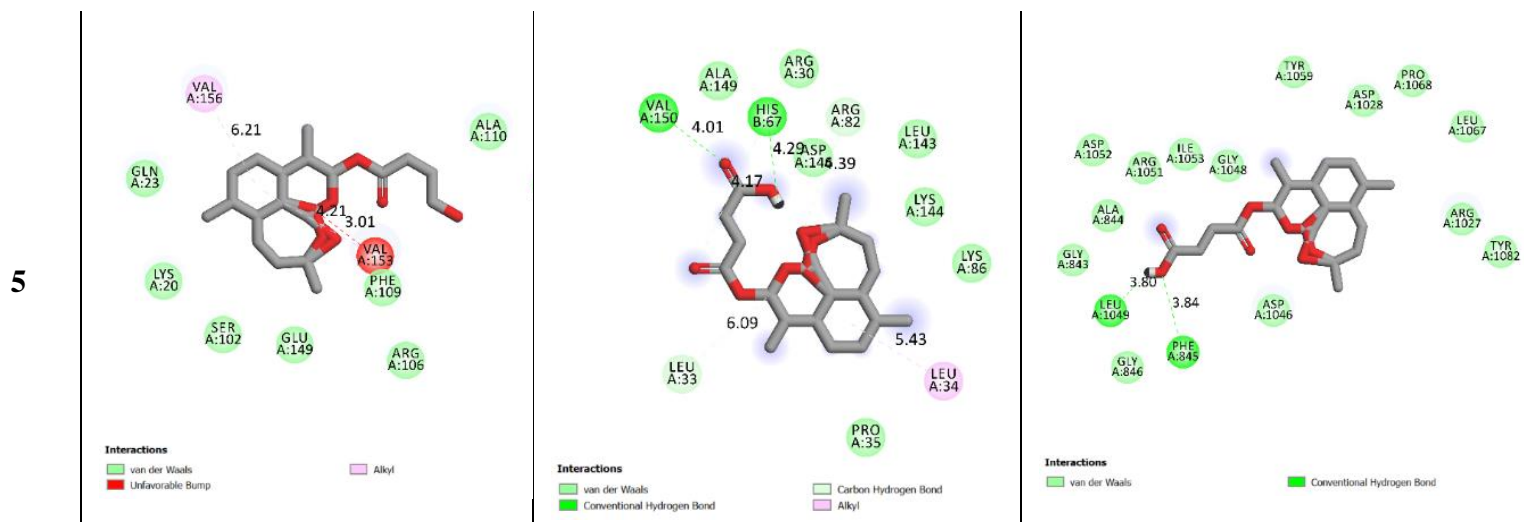

Hydrogen bonding was also evaluated for the interaction of Artemisinins 1-5 with these three targets. Table 4 summarizes the amino acid residues involved in hydrogen bonding of Artemisinins 1-5 within the binding sites of Bcl-2, CDK-6, and VEGFR-2. Total binding strength is a result of many types of bonds, including ionic, hydrophobic interactions, and Vander Waals forces, though hydrogen bonds being major contributors [50,51]. Hydrogen bonding also depends on the composition and 3D alignment of contacting amino acid residues at the prominent and active binding sites [52].

Table 4. Binding energies (Kcal/mol) and amino acid residues involved in hydrogen bonding of Artemisinins 15 within the binding sites of Bcl-2, CDK-6, and VEGFR-2.

\begin{tabular}{|c|c|c|c|}
\hline Drug & Targets & $\begin{array}{l}\text { Receptor residues involved in } \\
\text { Hydrogen bonding }\end{array}$ & $\begin{array}{c}\text { Binding energy } \\
(\text { Kcal/mol })\end{array}$ \\
\hline \multirow[t]{3}{*}{1} & Bcl-2 & - & -2.4 \\
\hline & CDK-6 & $\operatorname{Arg}^{22}, \operatorname{Ser}^{138}$ & -7.0 \\
\hline & VEGFR-2 & - & -6.8 \\
\hline \multirow[t]{3}{*}{2} & $\mathrm{Bcl}-2$ & - & -1.5 \\
\hline & CDK-6 & - & -6.5 \\
\hline & VEGFR-2 & $\operatorname{Arg}^{1050}, \operatorname{Arg}^{842}$ & -6.6 \\
\hline \multirow[t]{3}{*}{3} & Bcl-2 & - & -1.2 \\
\hline & CDK-6 & $\operatorname{Arg}^{82}$, Leu $^{34}$ & -5.7 \\
\hline & VEGFR-2 & $\operatorname{Arg} 1051$ & -6.4 \\
\hline \multirow[t]{3}{*}{4} & Bcl-2 & - & -0.8 \\
\hline & CDK-6 & Ser ${ }^{195}$ & -5.8 \\
\hline & VEGFR-2 & $\operatorname{Arg} 1051$ & -6.5 \\
\hline \multirow[t]{3}{*}{5} & Bcl-2 & - & -0.4 \\
\hline & CDK-6 & $\mathrm{His}^{67}, \mathrm{Val}^{150}$ & -7.1 \\
\hline & VEGFR-2 & Leu $^{1049}, \mathrm{Phe}^{845}$ & -7.5 \\
\hline
\end{tabular}

Figure 3 displays the interacting amino acid residues between investigated Artemisinins 1-5 and respective targets. Figure 3 reveals that all five Artemisinins 1-5 (orange sticks) used were found to bind to Bcl-2 with binding energies $-2.4,-1.5,-1.2,-0.8$, and $-0.4 \mathrm{Kcal} / \mathrm{mol}$, respectively (Figure 4).

These drugs were able to bind to the Bcl-2 enzyme firmly and, therefore, could possibly inhibit its function. The binding energies gave an insight into the affinity of the drug to the target, which was in the order $1>2>3>4>5$. The docking analysis of drugs within the active binding sites of targets are shown in Table 3. Hydrogen bonding was also evaluated for the interaction of Artemisinins 1-5 with targets.

Table 4 summarizes the amino acid residues involved in the hydrogen bonding of Artemisinins 1-5 within the binding sites of targets. Total binding strength is a result of many types of bonds, including ionic, hydrophobic interactions, and Vander Waals forces, though hydrogen bonds being major contributors $[50,51]$. 

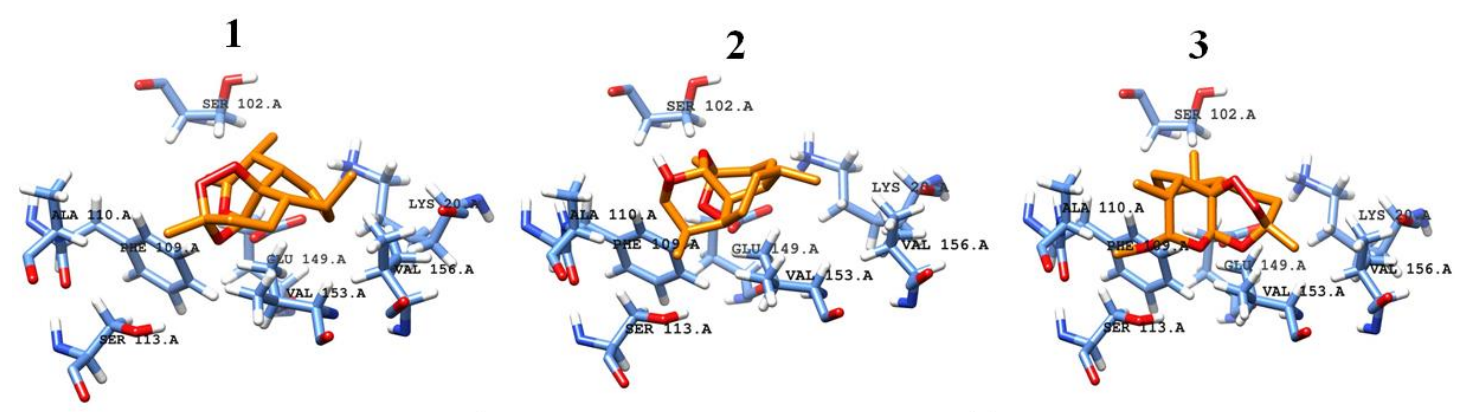

5

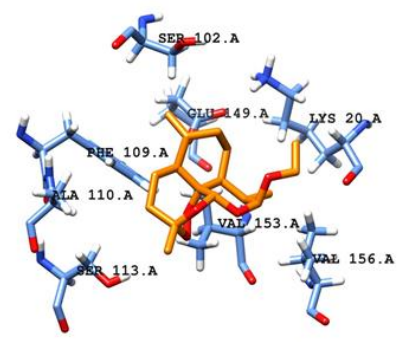

Bcl-2 (PDB ID: 2O2F)
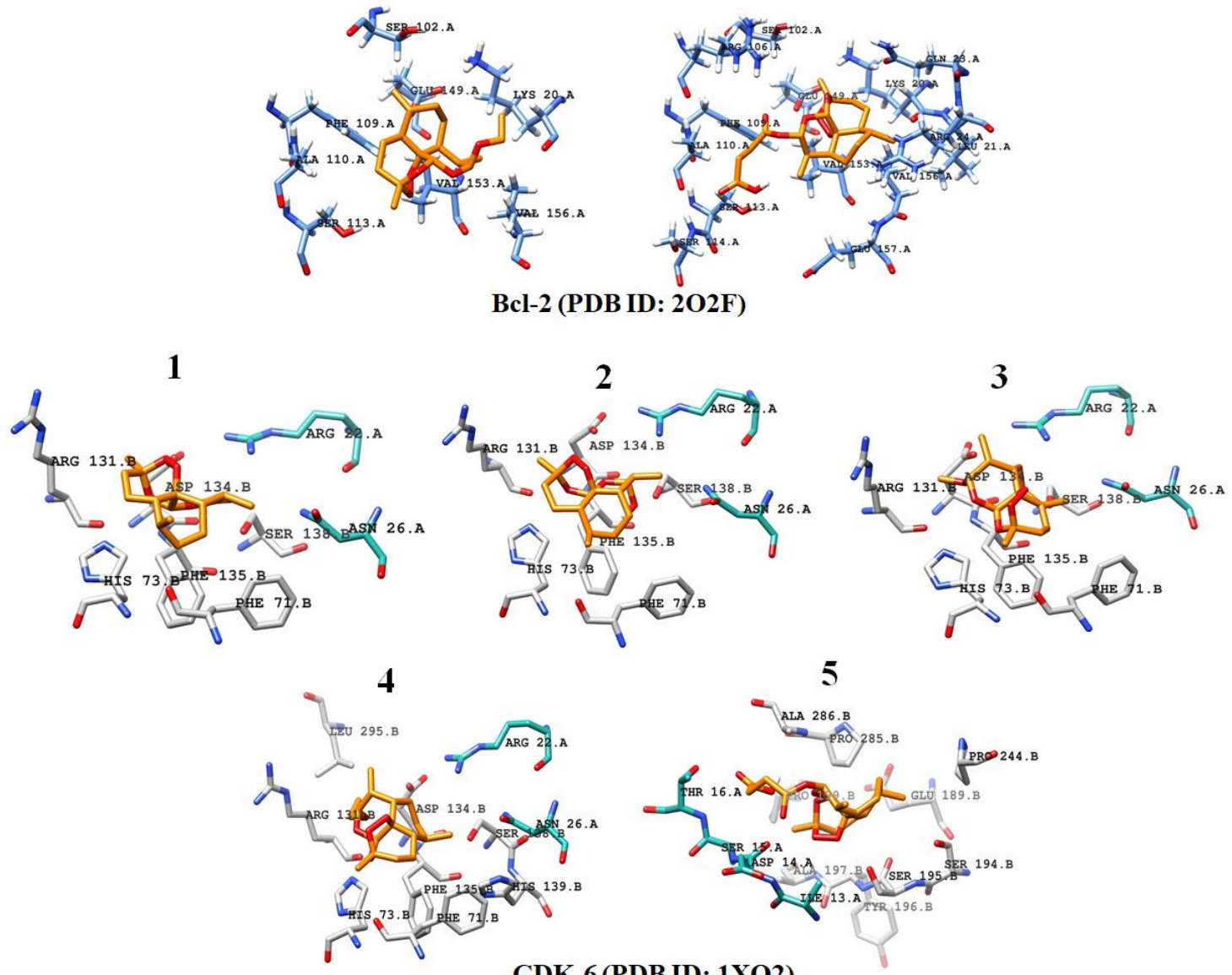

5

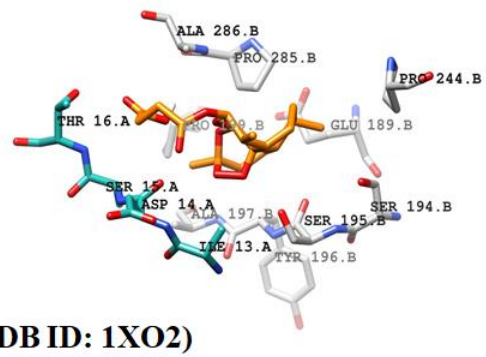

1

2
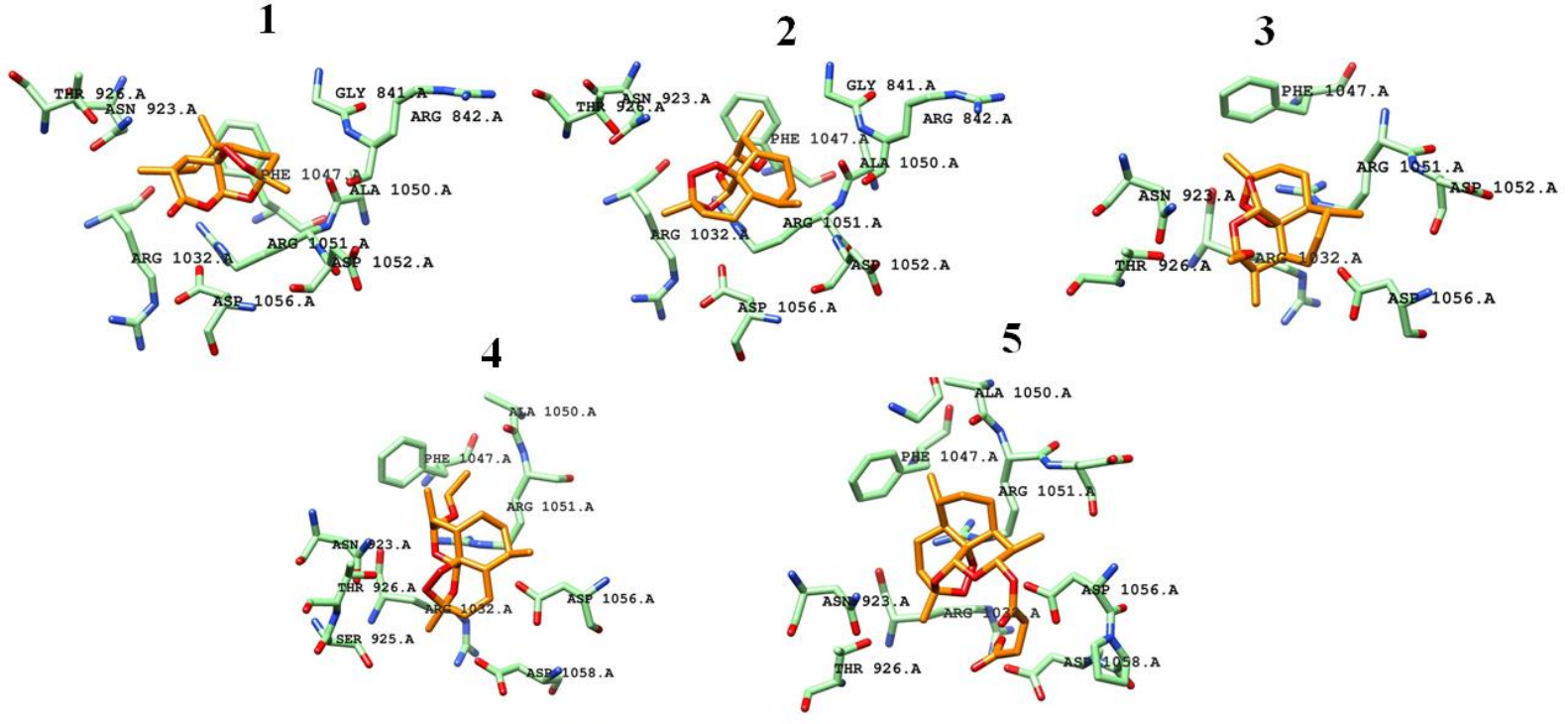

VEGFR-2 (PDB ID: 4ASD)

Figure 3. Alignment of the docked structures of Artemisinins 1-5 (orange stick) in the corresponding binding pockets of targets. 


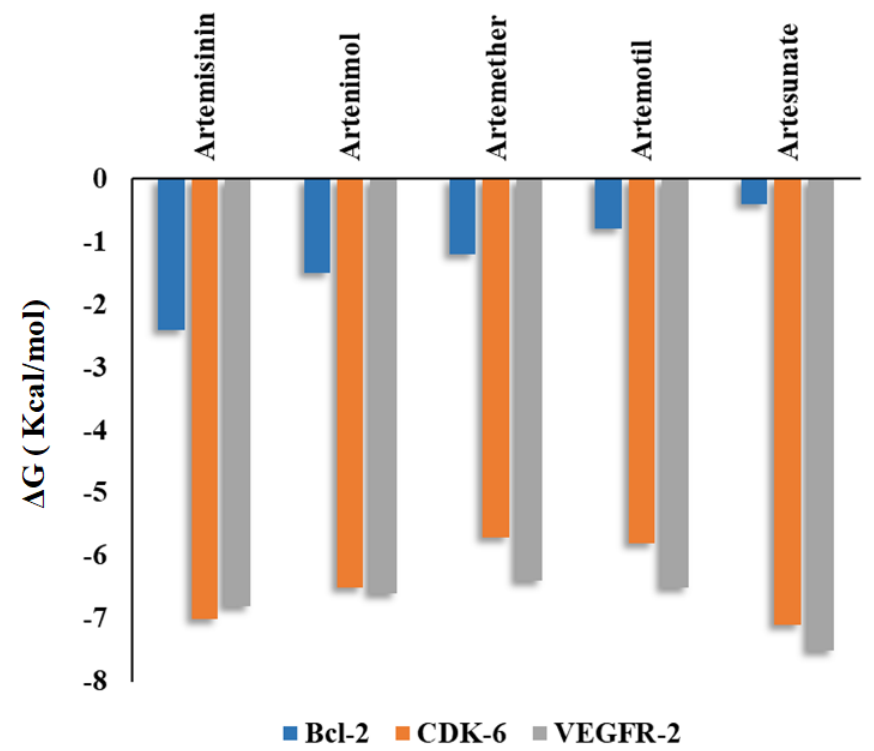

Figure 4. Bar graph representing the binding energies (Kcal/mol).

Hydrogen bonding also depends on the composition and 3D alignment of contacting amino acid residues at the prominent and active binding sites [52]. The results of docking analysis revealed that all the Artemisinins 1-5 were not capable of hydrogen bond formations with amino acid residues in the active site of the Bcl-2 enzyme (Tables 3 and 4). But the investigated drugs were involved in Vander Waals interactions with amino acid residues shown in Table 3.

With CDK-6, the Artemisinins 1-5 interacted with a greater binding affinity in comparison to Bcl-2. This was confirmed by the $\Delta \mathrm{G}$ values; $-7.0,-6.5,-5.7,-5.8,-7.1 \mathrm{Kcal} / \mathrm{mol}$, respectively with $1,2,3,4$ and 5 following the order $5>1>2>4>3$. The docking analysis exposed the hydrogen bonding interactions of investigated drugs with CDK-6. Artemisinin 1 formed a hydrogen bond with $\mathrm{Arg}^{22}, \operatorname{Ser}^{138}, 3$ with $\operatorname{Arg}^{82}$, Leu34, while 4 with $\operatorname{Ser}^{195}$ and 5 with $\mathrm{His}^{67}, \mathrm{Val}^{150}$. While drug 2 did not participate in hydrogen bonding (Tables 3 and 4).

The $\Delta \mathrm{G}$ values of the investigated Artemisinins 1-5 suggested that they interacted with the greatest binding affinity with the VEGFR-2 enzyme. The binding energies (Kcal $/ \mathrm{mol})$ were in the order $5(-7.5)>1(-6.8)>2(-6.6)>3(-6.5)>4(-6.4)$. The hydrogen-bonding interactions were with $\operatorname{Arg}^{1050}, \operatorname{Arg}^{842}$ (2), $\operatorname{Arg}^{1051}$ (3 and 4), and $\mathrm{Leu}^{1049}, \mathrm{Phe}^{845}$ (5), while no hydrogen bonds in the case of Artemisinins though other interactions were predicted.

From these findings, we could conclude that the variations due to the presence of contacting amino acid residues common to the active binding sites, with little relationship to the presence or absence of hydrogen bonds. This proposed that the presence of the hydrogen bonds was independent with the commonness of contacting amino acid residues to active binding sites and the strength of docking.

\subsection{DFT calculations.}

Density functional theory is a computer-based approach that has been gaining huge popularity in the field of in silico pharmaceutical analysis. It was performed to analyze the electronic and reactivity characteristics of Artemisinins 1-5. The analysis of the electronic characteristics of the ligands plays a central role in understanding their pharmacological properties. The DFT optimized structures of Artemisinins 1-5 are given in Figure 5. 

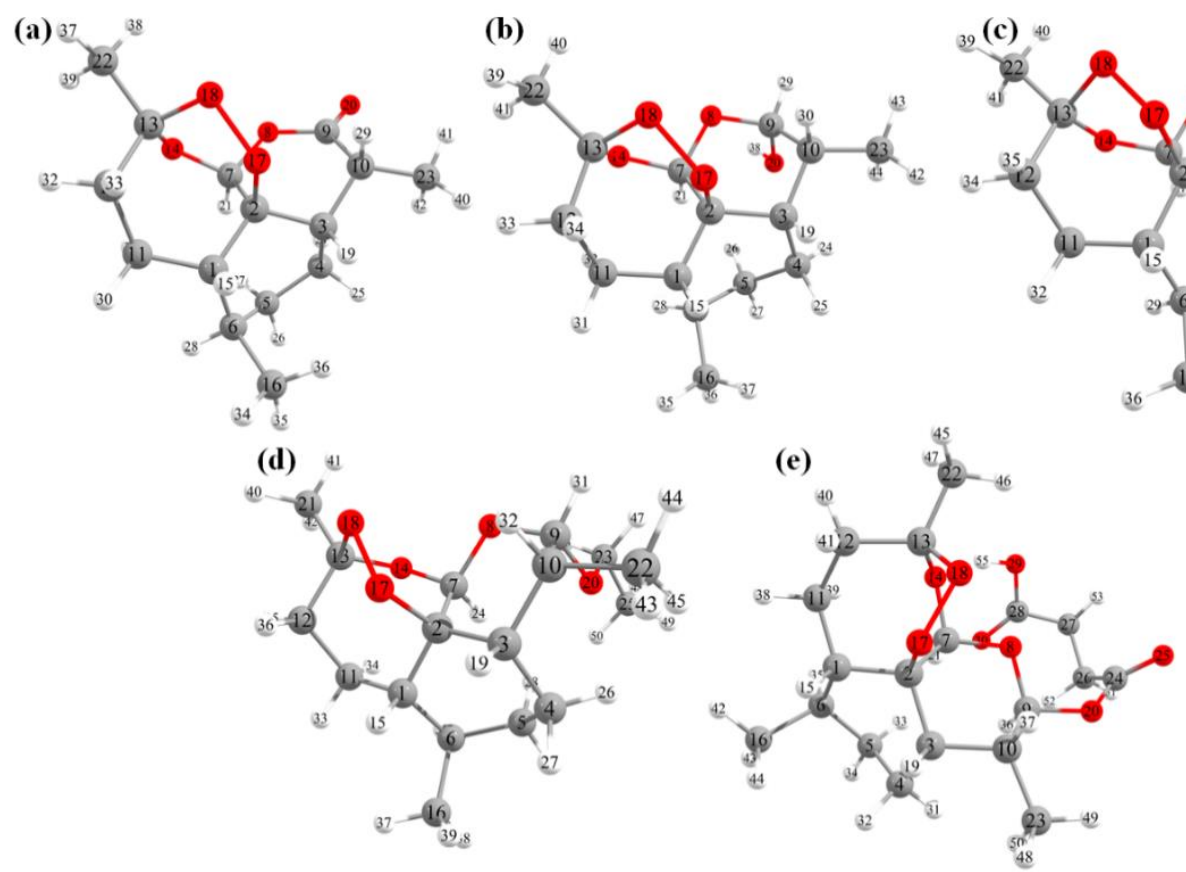

Figure 5. DFT-optimized geometry of (a) Artemisinin, (b) Artenimol, (c) Artemether, (d) Artemotil and (e) Artesunate.

Frontier molecular orbitals (FMOs) are the highest occupied molecular orbital (HOMO) and the lowest unoccupied molecular orbital (LUMO). The HOMO is the highest energy orbital occupied with electrons, so it is an electron donor, while LUMO is the lowest energy orbital that has a space to accept electrons, so it is an electron acceptor. These orbitals control the mode of the interaction of the drugs with other molecules, such as the interactions between these drugs and their receptors. Further, these drugs were analyzed on the basis of the HOMO/LUMO energy gap. As the gap energy increases, it leads to decrease reactivity and vice versa [53]. B3LYP functional method was applied for DFT calculations, while for calculating the band energy gap, $(\Delta \mathrm{E})$ expression of ELUмо-Eномо was used [54]. The results are summarized in Table 5.

Table 5. Thermal parameters (Hartree/Particle) (KJ/mol) and Dipole moment (Debye) of Artemisinins 1-5.

\begin{tabular}{c|c|c|c|c|c} 
Parameter & $\mathbf{1}$ & $\mathbf{2}$ & $\mathbf{3}$ & $\mathbf{4}$ & $\mathbf{5}$ \\
\hline ZPVE & 1008.251 & 1074.931 & 1153.743 & 1233.101 & 1304.262 \\
\hline $\mathrm{E}_{\text {tot }}$ & 1047.812 & 1114.751 & 1197.083 & 1279.621 & 1359.546 \\
\hline $\mathrm{H}$ & 1050.291 & 1117.230 & 1199.562 & 1282.100 & 1362.025 \\
\hline $\mathrm{G}$ & 903.812 & 970.774 & 1045.884 & 1121.247 & 1182.847 \\
\hline$\mu$ & 7.049131 & 2.342633 & 2.557229 & 2.675338 & 6.594904 \\
\hline
\end{tabular}

ZPVE: Sum of electronic and zero-point energies; $E_{\text {tot: }}$ Sum of electronic and thermal energies; $H$ : Sum of electronic and thermal enthalpies; G: Free energy; $\mu$ : Total Dipole Moment.

Figure 6 shows the HOMO and LUMO energies of the investigated artemisinins. The thermodynamic properties were also calculated and summarized in Table 5. The dipole moment in a molecule is another important electronic property. Whenever the molecule has a larger dipole moment, the intermolecular interactions are very strong [55]. The dipole moments of the investigated drugs were calculated to be in the order 1 (7.049131 Debye) $>5$ (6.594904 Debye $)>4(2.675338$ Debye $)>2(2.342633$ Debye $)>3(2.557229$ Debye $)$. These findings were in corroboration with the findings of molecular docking studies, suggesting that potent drug artemisinin 1 exhibited strong intermolecular interactions. 

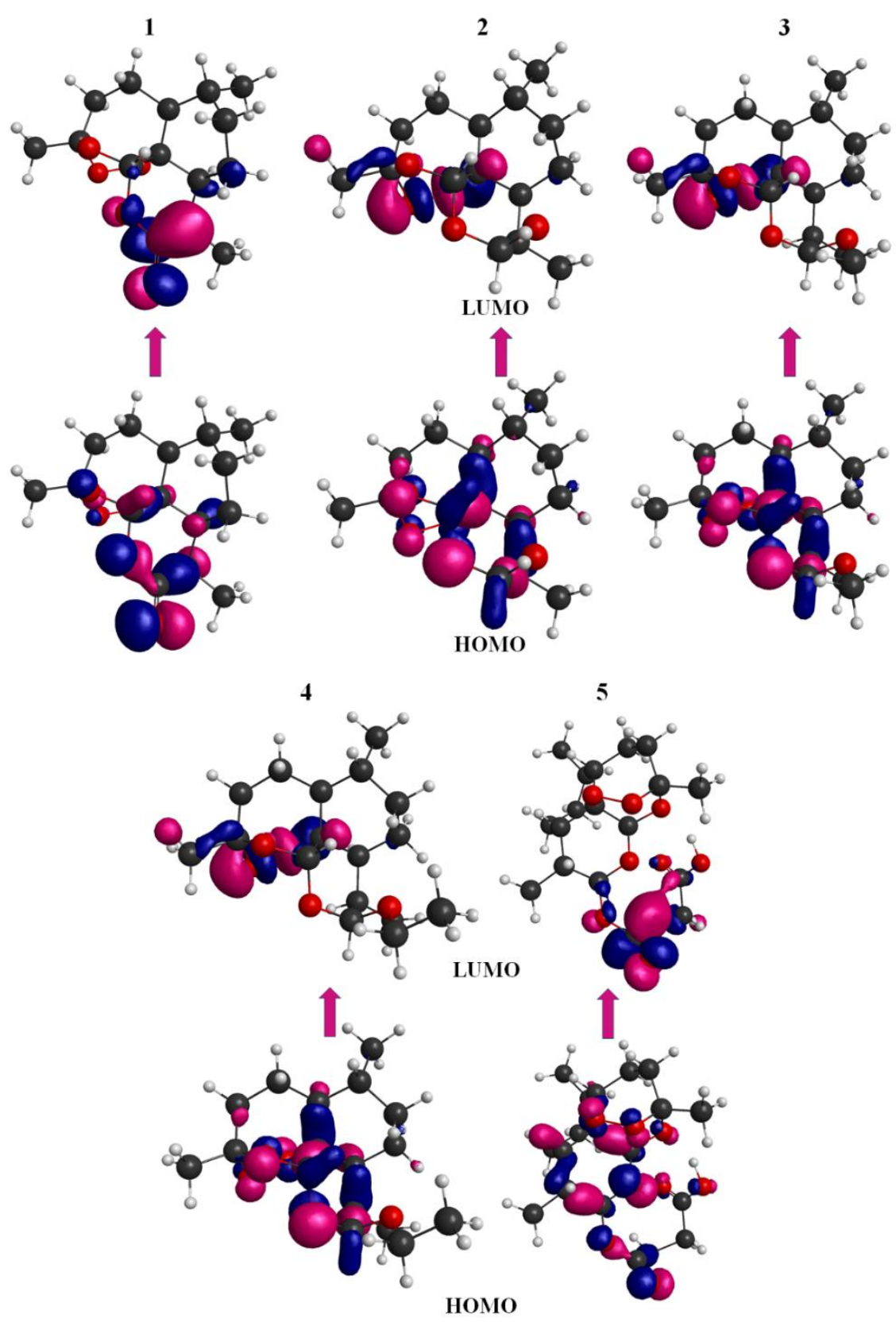

Figure 6. HOMO-LUMO energies of optimized structures of Artemisinins 1-5.

Besides the traditional reactivity descriptors (HOMO and LUMO), certain other chemical reactivity descriptors such as chemical hardness $(\eta)$, chemical softness $(\sigma)$, electrophilicity index $(\omega)$, nucleophilic index $(\varepsilon)$, electronegativity $(\chi)$, and chemical potential $(\mu)$ were also calculated and are summarized in Table 6 [38-40].

Table 6. The theoretical calculated conceptual DFT descriptors of Artemisinins 1-5.

\begin{tabular}{c|c|c|c|c|c} 
Parameters & $\mathbf{1}$ & $\mathbf{2}$ & $\mathbf{3}$ & $\mathbf{4}$ & $\mathbf{5}$ \\
\hline Eномо (a.u.) & -0.412 & -0.394 & -0.393 & -0.392 & -0.408 \\
\hline Elumo (a.u.) & 0.171 & 0.206 & 0.207 & 0.207 & 0.164 \\
\hline $\mathrm{Eg}$ (Hомо-Lumo) (a.u.) & -0.583 & -0.600 & -0.600 & -0.599 & -0.572 \\
\hline$\Delta$ E(Lumo-HOMO) & 0.583 & 0.600 & 0.600 & 0.599 & 0.572 \\
\hline$\chi$ (Electronegativity) & 0.1205 & 0.0940 & 0.0930 & 0.0925 & 0.1220 \\
\hline$\eta$ (Chemical hardness) & 0.2915 & 0.3000 & 0.3000 & 0.2995 & 0.2860 \\
\hline$\sigma$ (Chemical softness) & 3.430 & 3.333 & 3.333 & 3.338 & 3.496 \\
\hline$\mu$ (Chemical potential) & -0.1205 & -0.0940 & -0.0930 & -0.0925 & -0.1220 \\
\hline$\omega$ (Electrophilicity index) & -0.0711 & -0.0146 & -0.0143 & -0.0141 & -0.0246 \\
\hline$\varepsilon$ (Nucleophilic index) & -0.0351 & -0.0282 & -0.0279 & -0.0277 & -0.0348
\end{tabular}




\section{Conclusions}

Computational approaches are evolving day by day to improve the drug discovery process. The current study is based on drug repurposing of anti-malarial Artemisinins against anticancer chemotherapeutic targets viz., Bcl-2, CDK-6, and VEGFR-2. The ADME properties and drug-likeness of Artemisinins 1-5 (artemisinin, artenimol, artemether, artemotil, and artesunate) were also assessed, suggesting their good oral bioavailability. Antimalarial Artemisinins 1-5 were screened against Bcl-2, CDK-6, and VEGFR-2 through in silico approaches to find more potent inhibitors. In structure-based drug discovery, the binding site unlocks the active residues which participate in the interaction with the small molecule and undoubtedly essential for molecular docking. The detailed information about the binding pocket is mandatory for the structure-based drug discovery. The molecular docking study revealed the inhibitory effects of all five investigated artemisinins-based drugs. The binding energy $(\Delta \mathrm{G})$ values in $\mathrm{Kcal} / \mathrm{mol}$ gave an idea of the affinity of the binding of Artemisinins 1 5. The docking results revealed that all five Artemisinins were able to bind to CDK-6 and VEGFR-2 with more affinity in comparison to Bcl-2. Further, DFT calculations gave the knowledge of the electronic and structural properties as well as various reactivity descriptors. Thus, these anti-malarial drugs, viz., artemisinin, artenimol, artemether, artemotil, and artesunate, can be analyzed through the experiment in the future clinical trials of drugs against CDK-6 and VEGFR-2. However, it requires further study to elucidate this hypothesis.

\section{Funding}

This research received no external funding.

\section{Acknowledgments}

The author is grateful to Taibah University.

\section{Conflicts of Interest}

The authors declare no conflict of interest.

\section{References}

1. Rosenberg, S.A. Progress in human tumour immunology and immunotherapy. Nature 2001, 411, 380-384, https://doi.org/10.1038/35077246.

2. Cairns, J. The origin of human cancers. Nature 1981, 289, 353-357, https://doi.org/10.1038/289353a0.

3. Demaria, M.; O'Leary, M.N.; Chang, J.; Shao, L.; Liu, S.; Alimirah, F.; Koenig, K.; Le, C.; Mitin, N.; Deal, A.M.; Alston, S.; Academia, E.C.; Kilmarx, S.; Valdovinos, A.; Wang, B.; de Bruin, A.; Kennedy, B.K.; Melov, S.; Zhou, D.; Sharpless, N.E.; Muss, H.; Campisi, J. Cellular senescence promotes adverse effects of chemotherapy and cancer relapse. Cancer Discov. 2017, 7, 165-176, https://doi.org/10.1158/2159-8290.CD16-0241.

4. Mansoori, B.; Mohammadi, A.; Davudian, S.; Shirjang, S.; Baradaran, B. The Different Mechanisms of Cancer Drug Resistance: A Brief Review. Adv. Pharm. Bull. 2017, 7, 339-348, https://doi.org/10.15171/apb.2017.041.

5. Parvathaneni, V.; Kulkarni, N.S.; Muth, A.; Gupta, V. Drug repurposing: a promising tool to accelerate the drug discovery process. Drug Discov. Today 2019, 24, 2076-2085, https://doi.org/10.1016/j.drudis.2019.06.014.

6. Naylor, S.; Kauppi, D.M.; Schonfeld, J.M. Therapeutic drug repurposing, repositioning and rescue: Part II: Business review. Drug Discov. World 2015, 16, 57-72.

7. Seidel, T.; Wieder, O.; Garon, A.; Langer, T. Applications of the Pharmacophore Concept in Natural Product inspired Drug Design. Mol. Inform. 2020, https://doi.org/10.1002/minf.202000059.

8. Ho, W.E.; Peh, H.Y.; Chan, T.K.; Wong, W.S.F. Artemisinins: Pharmacological actions beyond anti- 
malarial. Pharmacol. Ther. 2014, 142, 126-139, https://doi.org/10.1016/j.pharmthera.2013.12.001.

9. Gao, F.; Sun, Z.; Kong, F.; Xiao, J. Artemisinin-derived hybrids and their anticancer activity. Eur. J. Med. Chem. 2020, 188, https://doi.org/10.1016/j.ejmech.2020.112044.

10. Li, R.; Li, Q.; Ji, Q. Molecular targeted study in tumors: From western medicine to active ingredients of traditional Chinese medicine. Biomed. Pharmacother. 2020, https://doi.org/10.1016/j.biopha.2019.109624.

11. Zhang, Y.; Zhang, M.; Wang, Y.; Fan, Y.; Chen, X.; Yang, Y.; Hua, Y.; Xie, W.; Lu, T.; Tang, W.; Chen, Y.; Liu, H. Protein-ligand interaction-guided discovery of novel VEGFR-2 inhibitors. J. Biomol. Struct. Dyn. 2020, 38, 2559-2574, https://doi.org/10.1080/07391102.2019.1635915.

12. Warren, C.F.A.; Wong-Brown, M.W.; Bowden, N.A. BCL-2 family isoforms in apoptosis and cancer. Cell Death Dis. 2019, 10, https://doi.org/10.1038/s41419-019-1407-6.

13. Ilizaliturri-Flores, I.; Correa-Basurto, J.; Bello, M.; Rosas-Trigueros, J.L.; Zamora-López, B.; BenítezCardoza, C.G.; Zamorano-Carrillo, A. Mapping the intrinsically disordered properties of the flexible loop domain of Bcl-2: a molecular dynamics simulation study. J. Mol. Model. 2016, 22, https://doi.org/10.1007/s00894-016-2940-1.

14. Verma, S.; Singh, A.; Mishra, A. Complex disruption effect of natural polyphenols on Bcl-2-Bax: molecular dynamics simulation and essential dynamics study. J. Biomol. Struct. Dyn. 2015, 33, 1094-1106, https://doi.org/10.1080/07391102.2014.931823.

15. Ilizaliturri-Flores, I.; Correa-Basurto, J.; Benítez-Cardoza, C.G.; Zamorano-Carrillo, A. A study of the structural properties and thermal stability of human Bcl-2 by molecular dynamics simulations. J. Biomol. Struct. Dyn. 2014, 32, 1707-1719, https://doi.org/10.1080/07391102.2013.833858.

16. Lim, S.; Kaldis, P. Cdks, cyclins and CKIs: roles beyond cell cycle regulation. Development 2013, 140, 3079-3093, https://doi.org/10.1242/dev.091744.

17. Tigan, A.-S.; Bellutti, F.; Kollmann, K.; Tebb, G.; Sexl, V. CDK6 - a review of the past and a glimpse into the future: from cell-cycle control to transcriptional regulation. Oncogene 2016, 35, 3083-3091, https://doi.org/10.1038/onc.2015.407.

18. Liu, Y.; Wang, X.; Wang, X.; Yu, R.; Liu, D.; Kang, C. De novo design of VEGFR-2 tyrosine kinase inhibitors based on a linked-fragment approach. J. Mol. Model. 2016, 22, https://doi.org/10.1007/s00894016-3088-8.

19. Malumbres, M.; Barbacid, M. To cycle or not to cycle: A critical decision in cancer. Nat. Rev. Cancer 2001, 1, 222-231, https://doi.org/10.1038/35106065.

20. Malumbres, M.; Barbacid, M. Cell cycle, CDKs and cancer: a changing paradigm. Nat. Rev. Cancer 2009, 9, 153-166, https://doi.org/10.1038/nrc2602.

21. Dash, R.; Junaid, M.; Mitra, S.; Arifuzzaman, M.; Hosen, S.M.Z. Structure-based identification of potent VEGFR-2 inhibitors from in vivo metabolites of a herbal ingredient. J. Mol. Model. 2019, 25, https://doi.org/10.1007/s00894-019-3979-6.

22. Sun, C.; Feng, L.; Sun, X.; Yu, R.; Kang, C. Design and screening of FAK, CDK 4/6 dual inhibitors by pharmacophore model, molecular docking, and molecular dynamics simulation. J. Biomol. Struct. Dyn. 2020, 1-10, https://doi.org/10.1080/07391102.2020.1786458.

23. Nazer, B.; Humphreys, B.D.; Moslehi, J. Effects of Novel Angiogenesis Inhibitors for the Treatment of Cancer on the Cardiovascular System. Circulation 2011, 124, 1687-1691, https://doi.org/10.1161/CIRCULATIONAHA.110.992230.

24. Gotink, K.J.; Verheul, H.M.W. Anti-angiogenic tyrosine kinase inhibitors: what is their mechanism of action? Angiogenesis 2010, 13, 1-14, https://doi.org/10.1007/s10456-009-9160-6.

25. Vittorio, S.; Seidel, T.; Germanò, M.P.; Gitto, R.; Ielo, L.; Garon, A.; Rapisarda, A.; Pace, V.; Langer, T.; De Luca, L. A Combination of Pharmacophore and Docking-based Virtual Screening to Discover new Tyrosinase Inhibitors. Mol. Inform. 2020, 39, https://doi.org/10.1002/minf.201900054.

26. Goel, H.L.; Mercurio, A.M. VEGF targets the tumour cell. Nat. Rev. Cancer 2013, 13, 871-882, https://doi.org/10.1038/nrc3627.

27. Huang, L.; Huang, Z.; Bai, Z.; Xie, R.; Sun, L.; Lin, K. Development and strategies of VEGFR-2/KDR inhibitors. Future Med. Chem. 2012, 4, 1839-1852, https://doi.org/10.4155/fmc.12.121.

28. Daina, A.; Michielin, O.; Zoete, V. SwissTargetPrediction: updated data and new features for efficient prediction of protein targets of small molecules. Nucleic Acids Res. 2019, 47, W357-W364, https://doi.org/10.1093/nar/gkz382.

29. Banerjee, P.; Eckert, A.O.; Schrey, A.K.; Preissner, R. ProTox-II: a webserver for the prediction of toxicity of chemicals. Nucleic Acids Res. 2018, 46, W257-W263, https://doi.org/10.1093/nar/gky318.

30. Lipinski, C.A.; Lombardo, F.; Dominy, B.W.; Feeney, P.J. Experimental and computational approaches to estimate solubility and permeability in drug discovery and development settings. Adv. Drug Deliv. Rev. 1997, 23, 3-25, https://doi.org/10.1016/S0169-409X(96)00423-1.

31. Trott, O.; Olson, A.J. AutoDock Vina: Improving the speed and accuracy of docking with a new scoring function, efficient optimization, and multithreading. J. Comput. Chem. 2009, https://doi.org/10.1002/jcc.21334.

32. Bruncko, M.; Oost, T.K.; Belli, B.A.; Ding, H.; Joseph, M.K.; Kunzer, A.; Martineau, D.; McClellan, W.J.; 
Mitten, M.; Ng, S.-C.; Nimmer, P.M.; Oltersdorf, T.; Park, C.-M.; Petros, A.M.; Shoemaker, A.R.; Song, X.; Wang, X.; Wendt, M.D.; Zhang, H.; Fesik, S.W.; Rosenberg, S.H.; Elmore, S.W. Studies Leading to Potent, Dual Inhibitors of Bcl-2 and Bcl-xL. J. Med. Chem. 2007, 50, 641-662, https://doi.org/10.1021/jm061152t.

33. Selvaraj, G.; Kaliamurthi, S.; Kaushik, A.C.; Khan, A.; Wei, Y.K.; Cho, W.C.; Gu, K.; Wei, D.Q. Identification of target gene and prognostic evaluation for lung adenocarcinoma using gene expression metaanalysis, network analysis and neural network algorithms. J. Biomed. Inform. 2018, 86, 120-134, https://doi.org/10.1016/j.jbi.2018.09.004.

34. McTigue, M.; Murray, B.W.; Chen, J.H.; Deng, Y.-L.; Solowiej, J.; Kania, R.S. Molecular conformations, interactions, and properties associated with drug efficiency and clinical performance among VEGFR TK inhibitors. Proc. Natl. Acad. Sci. 2012, 109, 18281-18289, https://doi.org/10.1073/pnas.1207759109.

35. Morris, G.M.; Huey, R.; Lindstrom, W.; Sanner, M.F.; Belew, R.K.; Goodsell, D.S.; Olson, A.J. AutoDock4 and AutoDockTools4: Automated docking with selective receptor flexibility. J. Comput. Chem. 2009, 30, 2785-2791, https://doi.org/10.1002/jcc.21256.

36. Schmidt, M.W.; Baldridge, K.K.; Boatz, J.A.; Elbert, S.T.; Gordon, M.S.; Jensen, J.H.; Koseki, S.; Matsunaga, N.; Nguyen, K.A.; Su, S.; Windus, T.L.; Dupuis, M.; Montgomery Jr, J.A. General atomic and molecular electronic structure system. J. Comput. Chem. 1993, 14, 1347-1363, https://doi.org/10.1002/jcc.540141112.

37. Gordon, M.S.; Schmidt, M.W. Advances in electronic structure theory. In: Theory and Applications of Computational Chemistry. Elsevier, 2005; pp. 1167-1189.

38. Shahab, S.; Sheikhi, M.; Filippovich, L.; Anatol'evich, D.E.; Yahyaei, H. Quantum chemical modeling of new derivatives of ( E,E )-azomethines: Synthesis, spectroscopic (FT-IR, UV/Vis, polarization) and thermophysical investigations. J. Mol. Struct. 2017, 1137, 335-348, https://doi.org/10.1016/j.molstruc.2017.02.056.

39. Shahab, S.; Filippovich, L.; Sheikhi, M.; Kumar, R.; Dikusar, E.; Yahyaei, H.; Muravsky, A. Polarization, excited states, trans-cis properties and anisotropy of thermal and electrical conductivity of the 4(phenyldiazenyl)aniline in PVA matrix. J. Mol. Struct. 2017, 1141, 703-709, https://doi.org/10.1016/j.molstruc.2017.04.014.

40. Shahab, S. Spectroscopic (Polarization, ExcitedState, FT-IR, UV/Vis and 1H NMR) and Thermophysical Investigations of New Synthesized Azo Dye and Its Application in Polarizing Film. Am. J. Mater. Synth. Process. 2017, 2, 17, https://doi.org/10.11648/j.ajmsp.20170202.11.

41. Cheng, W.; Yuan, Y.; Qiu, N.; Peng, P.; Sheng, R.; Hu, Y. Identification of novel 4-anilinoquinazoline derivatives as potent EGFR inhibitors both under normoxia and hypoxia. Bioorganic Med. Chem. 2014, 22, 6796-6805, https://doi.org/10.1016/j.bmc.2014.10.038.

42. Veber, D.F.; Johnson, S.R.; Cheng, H.-Y.; Smith, B.R.; Ward, K.W.; Kopple, K.D. Molecular Properties That Influence the Oral Bioavailability of Drug Candidates. J. Med. Chem. 2002, 45, 2615-2623, https://doi.org/10.1021/jm020017n.

43. Abdel-Mohsen, H.T.; Abood, A.; Flanagan, K.J.; Meindl, A.; Senge, M.O.; El Diwani, H.I. Synthesis, crystal structure, and ADME prediction studies of novel imidazopyrimidines as antibacterial and cytotoxic agents. Arch. Pharm. (Weinheim). 2020, 353, https://doi.org/10.1002/ardp.201900271.

44. Daina, A.; Michielin, O.; Zoete, V. SwissADME: A free web tool to evaluate pharmacokinetics, druglikeness and medicinal chemistry friendliness of small molecules. Sci. Rep. 2017, 7, https://doi.org/10.1038/srep42717.

45. Cereto-Massagué, A.; Ojeda, M.J.; Valls, C.; Mulero, M.; Pujadas, G.; Garcia-Vallve, S. Tools for in silico target fishing. Methods 2015, 71, 98-103, https://doi.org/10.1016/j.ymeth.2014.09.006.

46. Ding, H.; Takigawa, I.; Mamitsuka, H.; Zhu, S. Similarity-based machine learning methods for predicting drug-target interactions: a brief review. Brief. Bioinform. 2014, 15, 734-747, https://doi.org/10.1093/bib/bbt056.

47. Selvam, C.; Mock, C.D.; Mathew, O.P.; Ranganna, K.; Thilagavathi, R. Discovery of Vascular Endothelial Growth Factor Receptor-2 (VEGFR-2) Inhibitors by Ligand-based Virtual High Throughput Screening. Mol. Inform. 2020, https://doi.org/10.1002/minf.201900150.

48. Kojetin, D.J.; Burris, T.P. Small Molecule Modulation of Nuclear Receptor Conformational Dynamics: Implications for Function and Drug Discovery. Mol. Pharmacol. 2013, 83, 1-8, https://doi.org/10.1124/mol.112.079285.

49. Schena, A.; Griss, R.; Johnsson, K. Modulating protein activity using tethered ligands with mutually exclusive binding sites. Nat. Commun. 2015, 6, https://doi.org/10.1038/ncomms8830.

50. Papaneophytou, C.P.; Grigoroudis, A.I.; McInnes, C.; Kontopidis, G. Quantification of the Effects of Ionic Strength, Viscosity, and Hydrophobicity on Protein-Ligand Binding Affinity. ACS Med. Chem. Lett. 2014, 5, 931-936, https://doi.org/10.1021/ml500204e.

51. Zhao, X.; Xu, Z.; Li, H. NSAIDs Use and Reduced Metastasis in Cancer Patients: results from a metaanalysis. Sci. Rep. 2017, 7, https://doi.org/10.1038/s41598-017-01644-0.

52. Chelliah, V.; Blundell, T.L.; Fernández-Recio, J. Efficient Restraints for Protein-Protein Docking by Comparison of Observed Amino Acid Substitution Patterns with those Predicted from Local Environment. 
J. Mol. Biol. 2006, 357, 1669-1682, https://doi.org/10.1016/j.jmb.2006.01.001.

53. Zheng, Y.; Zheng, M.; Ling, X.; Liu, Y.; Xue, Y.; An, L.; Gu, N.; Jin, M. Design, synthesis, quantum chemical studies and biological activity evaluation of pyrazole-benzimidazole derivatives as potent Aurora A/B kinase inhibitors. Bioorg. Med. Chem. Lett. 2013, 23, 3523-3530, https://doi.org/10.1016/j.bmcl.2013.04.039.

54. Middleton, E.; Kandaswami, C.; Theoharides, T.C. The effects of plant flavonoids on mammalian cells: implications for inflammation, heart disease, and cancer. Pharmacol. Rev. 2000, 52, 673-751.

55. Xavier, S.; Periandy, S.; Ramalingam, S. NBO, conformational, NLO, HOMO-LUMO, NMR and electronic spectral study on 1-phenyl-1-propanol by quantum computational methods. Spectrochim. Acta Part A Mol. Biomol. Spectrosc. 2015, 137, 306-320, https://doi.org/10.1016/j.saa.2014.08.039. 